\title{
Selective Reactions of Cutaneous and Muscle Afferent Neurons to Peripheral Nerve Transection in Rats
}

\author{
Ping $\mathrm{Hu}^{1}$ and Elspeth M. McLachlan ${ }^{1,2}$ \\ ${ }^{1}$ Prince of Wales Medical Research Institute, and 2the University of New South Wales, Randwick, New South Wales 2031, Australia
}

To determine whether peripheral nerve injury has similar effects on all functional types of afferent neuron, we retrogradely labeled populations of neurons projecting to skin and to muscle with FluoroGold and lesioned various peripheral nerves in the rat. Labeled neurons were counted after different periods and related to immunohistochemically identified ectopic terminals and satellite cells in lumbar dorsal root ganglia.

After 10 weeks, $30 \%$ of cutaneous afferent somata labeled from transected sural nerves had disappeared but, if all other branches of the sciatic nerve had also been cut, $60 \%$ of cutaneous neurons were lost. Small-diameter sural neurons preferentially disappeared. In contrast, the number of muscle afferent somata was not affected by transection of various nerves. p75 was downregulated in axotomized cutaneous neurons but in not axotomized muscle neurons.

Conversely, p75 was upregulated in satellite cells around cutaneous but not muscle neurons. Consistent with this, perineuronal rings containing tyrosine hydroxylase, calcitonin gene-related peptide, galanin, or synaptophysin were formed preferentially around cutaneous neurons. Selective lesions of predominantly cutaneous nerves triggered the formation of rings, but none were detected after selective lesions of muscle nerves.

We conclude that cutaneous neurons are both more vulnerable and more associated with ectopic nerve terminals than muscle neurons in dorsal root ganglia after transection and ligation of peripheral nerves.

Key words: injury; death; sympathetic; sprouting; perineuronal rings; dorsal root ganglia; DRG

\section{Introduction}

After transection of a peripheral nerve, a proportion ( $~ 35 \%)$ of small-diameter sensory neurons in the dorsal root ganglion (DRG) progressively die (Jänig and McLachlan, 1984; Lisney, 1989; Tandrup et al., 2000). This type of cell death occurs relatively slowly after a sciatic lesion, but proceeds more rapidly and is quantitatively more severe if the spinal nerve is cut close to the DRG (Lekan et al., 1997; Vestergaard et al., 1997). Death is thought to occur by apoptosis (Groves et al., 1997). Many previous studies have focused on the problems of quantifying the numbers of remaining DRG somata, particularly because this is complicated by their atrophy (Aldskogius and Risling, 1981; Vestergaard et al., 1997). Although loss of small neurons is clearly greater than that of neurons with myelinated axons, it is not known whether one particular functional population of afferent neurons is more likely to die.

A diversity of other changes occurs within DRGs projecting in peripheral nerves in response to trauma and inflammation. These include changes in neuropeptide expression in both small- and large-diameter neuron somata (Hökfelt et al., 1994; Noguchi et

Received July 29, 2003; revised Sept. 20, 2003; accepted Sept. 24, 2003.

This work was supported by the Commonwealth Department of Health and Aged Care (Australia) and by the Motor Accidents Authority of New South Wales. We are grateful to Wilfid Jänig for his helpful comments on this manuscript.

Correspondence should be addressed to Prof. E. M. McLachlan, Prince of Wales Medical Research Institute, Gate 1, Barker Street, Randwick, New South Wales 2031, Australia. E-mail e.mclachlan@unsw.edu.au.

Copyright $\odot 2003$ Society for Neuroscience 0270-6474/03/2310559-09\$15.00/0 al., 1994), increased excitability of somata (Liu et al., 2002), and infiltration of macrophages and lymphocytes ( $\mathrm{Lu}$ and Richardson, 1993; Hu and McLachlan, 2002). In addition, varicose sympathetic and sensory terminals grow into the DRGs that contain injured sensory neurons and form perineuronal rings around individual large-diameter (presumed mechanosensitive) neurons (McLachlan et al., 1993; Ramer et al., 1999; $\mathrm{Hu}$ and McLachlan, 2000; Li and Zhou, 2001). Although sympathetic sprouting depends on upregulation of neurotrophins (Zhou et al., 1999) and cytokines (Ramer et al., 1998), it is not clear whether sprouting occurs because of injury of neurons with particular targets or is simply related to the number of axotomized neurons.

Only one feature of lesioned DRGs has been identified in terms of which functional type of afferent is affected. Ectopic spontaneous activity is confined to a population of mediumdiameter muscle afferent neurons with myelinated axons (Michaelis et al., 2000). In these neurons, spontaneous subthreshold oscillations sufficient to reach threshold for action potentials develop more readily than in cutaneous afferent neurons (Liu et al., 2002).

In the present study, experiments were designed to test (1) whether the neurons that die after nerve lesions are those that originally supplied skin or muscle, (2) whether perineuronal rings form selectively around either cutaneous or muscle neurons, and (3) whether lesions to nerves that predominantly supply either skin or muscle are equally effective in triggering the 
Table 1. Antibodies used in this study

\begin{tabular}{|c|c|c|c|c|}
\hline $\begin{array}{l}\text { Anti- } \\
\text { body } \\
\text { to }\end{array}$ & Raised in & Company & Dilution with & Antibody to \\
\hline CGRP & Rabbit & Peninsula ${ }^{a}$ & 1:1000 & TH \\
\hline CGRP & Goat & Biogenesis $^{b}$ & 1:500 & p75 \\
\hline GAL & Rabbit & Peninsula ${ }^{a}$ & $1: 2000$ & Synaptophysin \\
\hline GAL & Rabbit & Peninsula $^{a}$ & $1: 2000$ & TH \\
\hline TH & Rabbit & Pelfreez ${ }^{c}$ & $1: 200$ & Synaptophysin \\
\hline GFAP & Rabbit & $\mathrm{Dako}^{d}$ & $1: 1000$ & CGRP \\
\hline p75 & Rabbit & Chemicon $^{e}$ & $1: 3000$ & Major histocompatibility complex class II \\
\hline
\end{tabular}

${ }^{a}$ Peninsula Laboratories, San Carlos, CA.

${ }^{b}$ Biogenesis Ltd, Poole, UK.

'Pelfreez, Rogers, AR.

${ }^{d}$ Dako, Glostrup, Denmark.

${ }^{e}$ Chemicon, Temecula, CA.

formation of perineuronal rings. The effects of sciatic and spinal nerve transections were examined in lumbar DRGs of Wistar rats in which these responses to nerve lesions are well documented.

\section{Materials and Methods}

Adult female Wistar rats (135-200 gm; 6-8 weeks of age at operation) were anesthetized with ketamine $(60 \mathrm{mg} / \mathrm{kg})$ and xylazine $(10 \mathrm{mg} / \mathrm{kg})$ administered together intraperitoneally for each nerve intervention. After surgery as described below, the wounds were treated with tetracycline powder and sealed with Michel clips. In each group of rats indicated below, the animals were held for survival periods of either 1 week or 8-12 weeks after the lesion unless indicated otherwise. The longer period is referred to as " 10 weeks" throughout this report for simplicity, reflecting the mean postoperative period of $9.8 \pm 0.2$ weeks. The animals were then deeply anesthetized with sodium pentobarbitone $(80 \mathrm{mg} / \mathrm{kg}$, i.p. $)$ and perfused through the descending aorta, first with heparinized $0.9 \% \mathrm{NaCl}$ containing $0.1 \% \mathrm{NaNO}_{2}$ and then with Zamboni's fixative. All procedures were approved by the University of New South Wales Animal Care and Ethics Committee. Every effort was made to minimize the suffering of the animals and the number of animals used.

Pairs of ipsilateral and contralateral L4 and L5 DRGs, with spinal roots and spinal nerves attached, and $10-15 \mathrm{~mm}$ lengths of the peripheral nerve distally down to the lesion site itself, were blocked and cut together, in some cases in the same blocks as corresponding tissue from an agematched naive control animal. The tissue was postfixed in fixative overnight, washed, and infiltrated with $30 \%$ sucrose before being frozen and stored at $-80^{\circ} \mathrm{C}$.

Retrograde tracing with FluoroGold. Before experimental nerve lesions, skin and muscle neurons were labeled in vivo using two different techniques: (1) to identify cutaneous afferent neurons, the left sural nerve was ligated and cut $2-3 \mathrm{~mm}$ above the ankle, and $\sim 2 \mu \mathrm{l}$ of $2 \%$ FluoroGold (FG) solution in $0.9 \% \mathrm{NaCl}$ was applied to the cut axons and held in contact with them in a small pledget of hemostatic foam fixed in place inside a short piece of polyvinylchloride tubing, as described in detail previously (Wesselmann and McLachlan, 1984). This technique was used rather than injection into a region of skin to provide more reproducible data. This means that comparisons could not be made with undamaged sural afferent neurons (compare muscle neurons, below).

(2) To label muscle neurons, $2-3 \mu \mathrm{l}$ of $2 \%$ FG solution was injected into both the anterior and posterior compartments of both distal hindlimbs (i.e., into medial and lateral gastrocnemius and tibialis anterior muscles). Injections were made over $5 \mathrm{~min}$ at three or four different sites in each muscle compartment via a number 27 needle that was left in place for $1 \mathrm{~min}$ after introducing each aliquot of FG to allow the label to dissipate through the muscle. The muscle surface was washed and dried carefully before closing the skin.

Nerve lesions. Groups of animals were studied after different nerve lesions in addition to the labeling procedures above: (1) after applying FG to the cut sural nerve, the animals were allowed to survive for 1 or 10 weeks before being perfused. (2) After applying FG to the cut sural nerve, the peroneal and tibial branches of the sciatic nerve were ligated and cut during the same operation (this is equivalent to transection of the entire sciatic nerve and will be referred as "transected sciatic"); these animals were allowed to survive for 10 weeks. (3) After injection of FG into the muscles on both sides of the animal, no nerve lesions were made; these animals were allowed to recover for 1 week. (4) One week after injection of FG into the muscles on both sides of the animal, the entire left sciatic nerve was ligated and cut just above its trifurcation; these animals were allowed to survive for 1 or 10 weeks. (5) One week after injection of FG into the muscles on both sides of the animal, the nerves to lateral gastrocnemius/soleus and to medial gastrocnemius and the muscle branches of the peroneal nerve projecting to tibialis anterior and other muscles of the anterior compartment of the lower leg were ligated and cut on the left side ("muscle nerves cut"); these animals were allowed to survive for 1 week. In this group the muscle neurons are axotomized for comparison with group 1. (6) One week after injection of FG into muscles on both sides of the animal, $\sim 2 \mathrm{~mm}$ of the left $\mathrm{L} 5$ spinal nerve $5-10 \mathrm{~mm}$ from its DRG was excised after removing the transverse process of L6; these animals were allowed to survive for 10 weeks. (7) The following largely cutaneous nerves were ligated and cut on the left side: the sural, superficial peroneal, and tibial nerves close to the ankle and the saphenous nerve at mid-thigh, all in the same animal ("skin nerves cut"); these animals were allowed to survive for 10 weeks to test the effect on the number of perineuronal rings of nerve terminals. (8) The above muscle nerves (see group 5) were ligated and cut on the left side (without injecting FG); these animals were allowed to survive for 10 weeks also to test the effect on the number of perineuronal rings.

It is important to note that there was no difference in the extent of the lesions to the labeled populations projecting to skin and muscle in groups 2 and 4 , because in each case the axons were only cut once.

Immunohistochemistry. Serial longitudinal cryostat sections (14 $\mu \mathrm{m})$ through entire DRGs were mounted sequentially across four slides (i.e., four sections per slide with sections $56 \mu \mathrm{m}$ apart) and stained immunohistochemically as described previously (McLachlan et al., 1994).

To demonstrate antigens in neuron somata, glia, axons, and terminals, fluorescent double-labeling techniques were applied using various combinations of antibodies as shown in Table 1. Briefly, after washing in 50\% ethanol, normally two sets of three adjacent slides from the middle of the DRGs were incubated in mixtures of two primary antibodies followed by CY3- and FITC-labeled secondary antibodies to IgG as appropriate. When sections containing FG-labeled neuron somata were studied, somatic antigens were demonstrated using a CY3-labeled secondary antibody to avoid any leak-through of FG fluorescence. Sections were visualized by epifluorescence using Leitz water-immersion objectives and Ploempak filters A (for FG), L4 (for FITC), and N2 (for CY3) and photographed on Tmax 400 film.

Quantitative evaluation. Immunoreactivity is indicated by a plus sign. Neuronal profiles containing nuclei were counted in four to eight sections selected at $224 \mu \mathrm{m}$ intervals through the middle of each DRG (one per slide). Sections of control DRGs contained $\sim 226$ neuronal profiles 

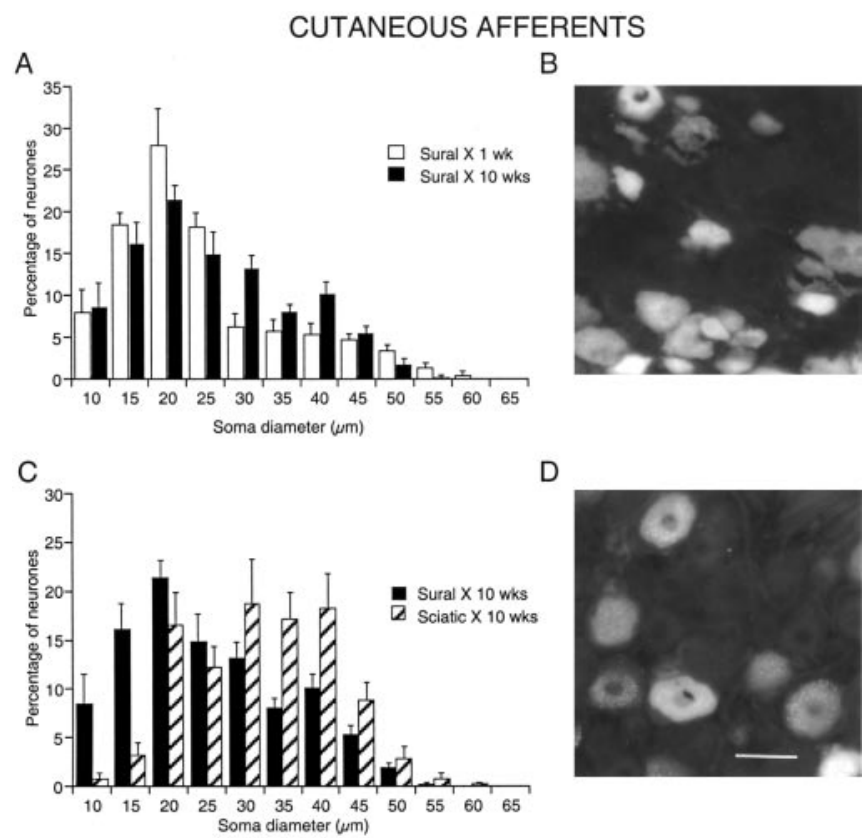

Figure 1. Loss of small-diameter cutaneous afferent neurons in L5 DRGs after sciatic nerve transection. Somata labeled retrogradely with $\mathrm{FG}$ applied to the cut axons of the left sural nerve. $A, C$, Histograms of soma diameter after nerve transection and ligation. Sural $X, 1$ wk, One week after cutting and labeling the sural nerve (illustrated in $B$ ). Sural $X, 10$ wks, Ten weeks after cutting and labeling the sural nerve. Sciatic $X, 10$ wks, Ten weeks after cutting and labeling the sural nerve and also cutting and ligating the tibial and peroneal branches of the sciatic nerve at the same operation (illustrated in D). Scale bar: (in D) $A-D, 50 \mu \mathrm{m}$.

containing nuclei, whereas they contained $\sim 195$ profiles 10 weeks after sciatic nerve transection and $\sim 125$ profiles 10 weeks after spinal nerve transection (Hu and McLachlan, 2001). This suggests that $\sim 13 \%$ of neurons were lost 10 weeks after sciatic nerve transection, which is slightly fewer than predicted from the results of similar experiments using stereological techniques (Tandrup et al., 2000). Such techniques are difficult to apply in the fluorescent microscope.

The average number of labeled neuron profiles per section was determined from 4-12 sections in each animal. No attempt was made to correct for double counting so that large neurons will have been sampled more frequently, but this is unlikely to affect the conclusions. Soma diameters were measured down the microscope as the average between long and short diameters of neuronal profiles (measured to the nearest 5 $\mu \mathrm{m})$. Normally $50-200$ somata were measured from each animal in each group.

Perineuronal baskets or "rings" were identified as immunoreactive axon terminals that surrounded $\geq 50 \%$ of a cell body circumference. These axons lay immediately adjacent to the soma or more commonly among the surrounding glia.

Data are expressed as mean \pm SEM throughout unless specified otherwise. Significant differences between groups of data were determined using an ANOVA with Fisher's protected least significant difference post hoc or Student's $t$ test, as appropriate. The $\chi^{2}$ test was used to determine whether significant changes in the proportions of neurons in different groups had occurred. $p<0.05$ was taken as significant.

\section{Results}

Approximately $60 \%$ of sural neurons had disappeared by 10 weeks after sciatic nerve transection

One week after application of FG to the cut sural nerve, $82 \pm 8$ brightly labeled neuron profiles were present in each section (Fig. $1 B$ ). After 10 weeks, there were only $58 \pm 4$ cells per section (Fig. $2 A)$, representing a significant reduction of $29 \%(p=0.014)$.

The mean diameter of labeled somata was not different between 1 and 10 weeks after sural transection $(p=0.25)$ (Fig. $2 B$ ).
A

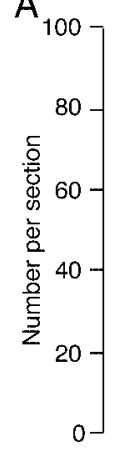

SURAL FG+

B
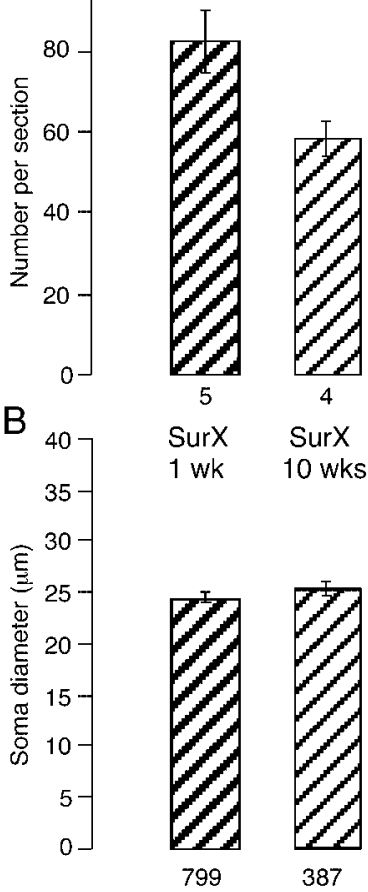

4

SurX

10 wks
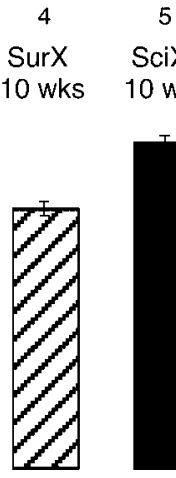

387

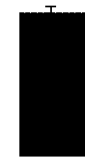

5

SciX

10 wks

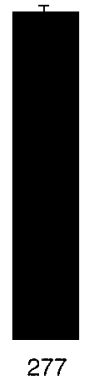

Figure 2. Number and size of neurons labeled from the sural nerve after different lesions. Somata labeled retrogradely $\mathrm{FG}+$ from the cut sural nerve. Histograms show the number of labeled somata in each section $(A)$ and their mean diameter $(B)$. The lesions are indicated between the plots together with the time in weeks (wks) since the lesion: SurX, only sural nerve cut; SciX, sciatic nerve cut. The numbers below each column indicate the number of animals (in $A$ ) and the number of neuronal profiles (in $B$ ). The number of sural neurons declined more if the entire sciatic nerve was cut; as mean diameter increased, small neurons had disappeared.

However, the distribution of cell diameters was changed such that the peak at $\sim 25 \mu \mathrm{m}$ was reduced by 10 weeks after the lesion (Fig. $1 A$ ), and there were significantly fewer neurons $<30 \mu \mathrm{m}$ in diameter $(62 \%)$ than at 1 week $(73 \% ; p=0.013)$.

When the peroneal and tibial nerves had also been cut at the time of sural nerve labeling, only $35 \pm 2$ neurons were labeled per section after 10 weeks (Figs. 1D, 2A). This reduction of $58 \%$ was highly significant $(p<0.001)$, representing a further loss of $40 \%$ of those cells present after 10 weeks when only the sural nerve had been cut, i.e., cutting the other axons in the sciatic nerve had an additional adverse effect on sural neurons. The distribution of soma diameters became markedly skewed to larger diameters (Fig. 1C) with mean cell diameter $30 \%$ larger (Fig. $2 B$ ) and the proportion of neurons $<30 \mu \mathrm{m}$ diameter $34 \%$ smaller than after sural axotomy alone ( $p<0.0001$ for both measures). The brightness of the remaining neurons was unchanged.

\section{Muscle afferent neurons did not die after axotomy by} transection of either the sciatic or spinal nerve

In animals fixed 1 week after injection of FG into both muscle compartments of the lower limb without lesioning any nerve, $9 \pm$ 2 labeled neuronal profiles (see Fig. $4 A$ ) were identified per section of L5 DRG. FG in labeled muscle somata commonly appeared as a cluster of fine bright granules adjacent to the nucleus (Fig. 3B) and was generally fainter than in somata labeled from cut sural axons (Fig. $1 B$ ). Labeled muscle afferent neuron profiles ranged widely in size, but few were $<20 \mu \mathrm{m}$ in diameter ( $\leq 5 \%$, compare $\sim 25 \%$ of sural neurons) (Fig. $3 A$, compare Fig. $1 A$ )

When the whole sciatic nerve was cut 1 week after the injection 


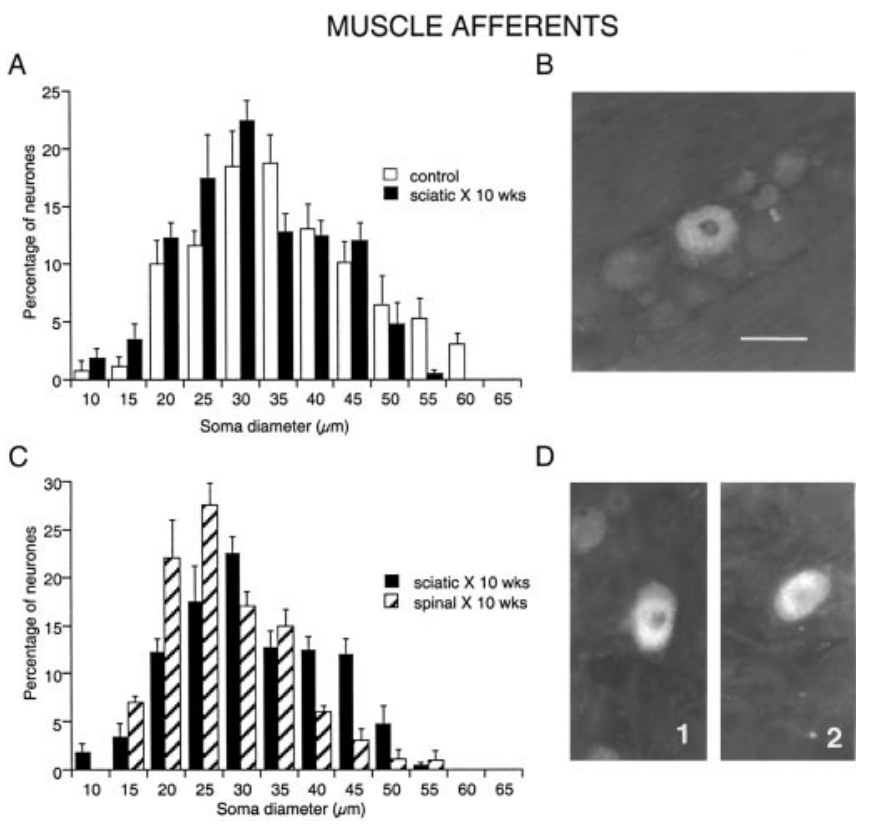

Figure 3. Changes in diameter of muscle afferent neurons in L5 DRGs after peripheral nerve transection. Somata labeled retrogradely with FG injected into the muscles of the lower hindlimb. $A, C$, Histograms of soma diameter with and without nerve transection and ligation. Control, One week after muscle injections with no nerve lesion (illustrated in $B$ ). Sciatic $X, 10$ wks, Ten weeks after cutting and ligating the sciatic nerve 1 week after muscle injections (illustrated in D1). Spinal X, 10 wks, Ten weeks after cutting and ligating the spinal nerve 1 week after muscle injections (illustrated in D2). Scale bar: (in B) $A-D, 50 \mu \mathrm{m}$.

of tracer, the number of labeled cells per section after a further week was not lower than in unlesioned animals (Fig. 4) $(p>0.2)$. However their brightness was enhanced, and the cells were more completely filled with FG, making them easier to detect. Contralateral to the sciatic lesions, the number of labeled neuron somata in L5 DRG was not different from the number on the operated side $(p=0.47)$. At 10 weeks after sciatic transection, again the FG + cells were brighter than contralaterally or in unlesioned controls (Fig. 3D1). However, the mean number per section $(9 \pm 1)$ was similar to that in either ipsilateral or contralateral ganglia 1 week after nerve transection or in naive controls (Fig. 4A) ( $p>0.2)$.

In another set of experiments, the nerves that supplied the injected muscles were selectively transected on the left side 1 week after intramuscular injections of FG. After a further week, the muscle afferent somata were again more brightly labeled on the ipsilateral than on the contralateral side, and the number per section $(10 \pm 1)$ was indistinguishable from that after the other lesions (Fig. $4 A$ ) or contralaterally.

Overall, the mean number of labeled muscle neurons in lesioned DRGs tended to be higher than for the uninjured DRGs $(p=0.07)$. This difference was significant only if a paired $t$ test between operated and contralateral DRGs was used ( $p=0.013$ ), although the validity of using a paired test given the nature of the muscle injections is doubtful. The enhanced brightness of FG labeling ipsilateral to the nerve lesion might reflect a reduced clearance of lysosomal particles or perhaps enhanced retrograde transport of the dye in axotomized neurons. It seems unlikely that the low counts in controls could have masked any decrease in muscle neurons on the injured side. Even if this had been so, there was no difference between axotomy (cutting the muscle nerves) and sciatic transection. This result is very different from that following sural nerve transection.

\section{A \\ MUSCLE FG+}
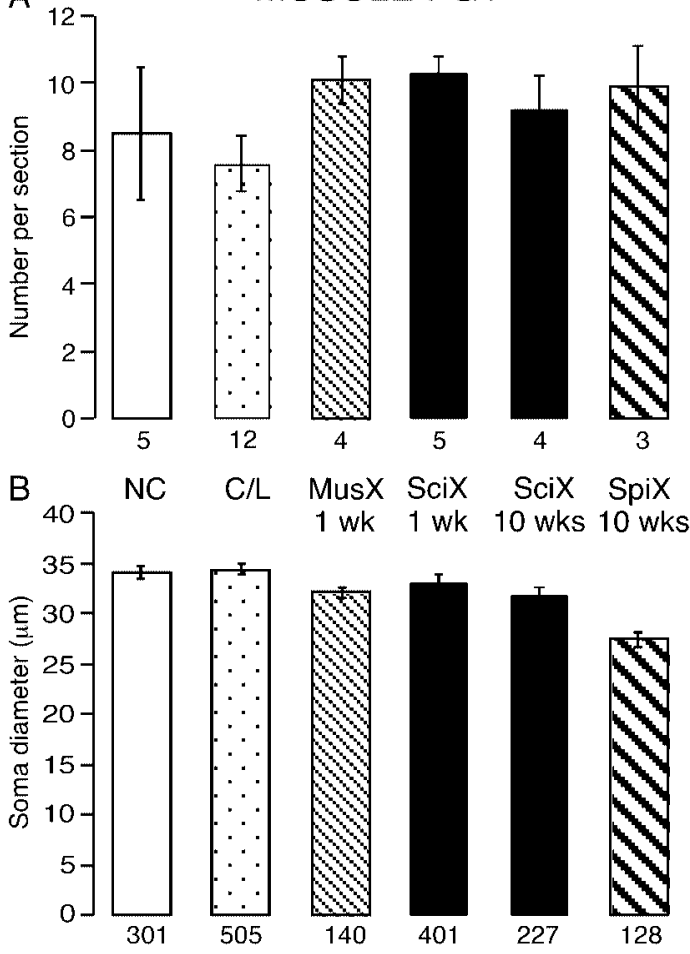

Figure 4. Number and size of neurons labeled from muscles after different lesions. Somata labeled retrogradely with $\mathrm{FG}+$ from injections into muscles of the lower hindlimb. Histograms show the number of labeled somata in each section $(A)$ and their mean diameter $(B)$. The lesions are indicated between the plots together with the time in weeks (wks) since the lesion: NC, naive control; C/I, contralateral to the lesions at 1 and 10 weeks (pooled); MusX, muscle nerves cut; SciX, sciatic nerve cut; SpiX, L5 spinal nerve cut. The numbers below each column indicate the number of animals (in $A$ ) and the number of neuronal profiles (in $B$ ). There was no significant loss of muscle afferents after any lesion. The cells atrophied slightly after spinal nerve transection.

The mean diameters of labeled muscle neurons 1 and 10 weeks after a sciatic transection were not different from each other $(p=$ 0.75 ) but were both significantly smaller than in uninjured controls $(p=0.01)$ (Fig. $4 \mathrm{~B})$. Thus, the $6 \%$ reduction in mean diameter that had occurred during the first week after axotomy did not change in the subsequent weeks. Overall, however, the size distributions for lesioned, naïve, and contralateral DRGs were indistinguishable ( $\chi^{2}$ test), even when all control data were pooled.

A more provocative test of the vulnerability of muscle afferent neurons to axotomy was to cut the L5 spinal nerve 1 week after injecting FG into the two muscle compartments. After a further 10 weeks, there was again a similar number of labeled neurons in L5 DRG as at either time point after a sciatic lesion $(p=0.93$ and 0.80 respectively) or in naive controls ( $p=0.55$ ) (Fig. $4 A$ ). Again the somata were more brightly labeled than on the contralateral side (Fig. 3D2). The number of labeled neurons detected contralateral to the spinal nerve transection $(6.5 \pm 2.4$ per section $)$ was not significantly different from that on the lesioned side ( $p=$ $0.17)$, that contralateral to the sciatic lesions $(p=0.53)$, or the naive controls $(p=0.34)$. Thus, cutting the axons of labeled muscle neurons closer to the soma and axotomizing almost all DRG neurons also did not lead to any reduction in the number of surviving cells. This indicates that afferent neurons labeled from their terminals in muscle are resistant both to axotomy and to the process that produces delayed cell death after lesions to other DRG neurons. 
TH
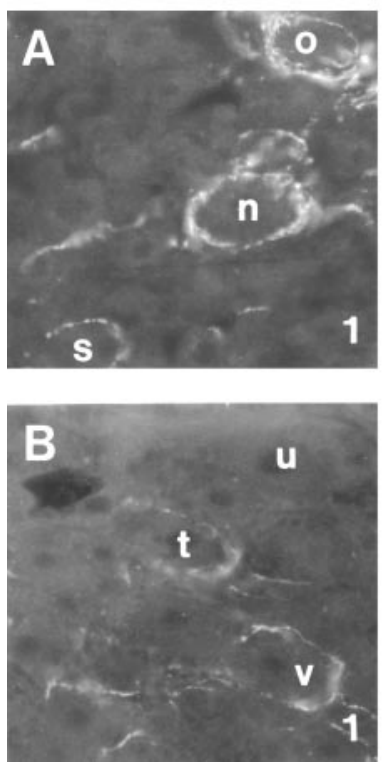

FG
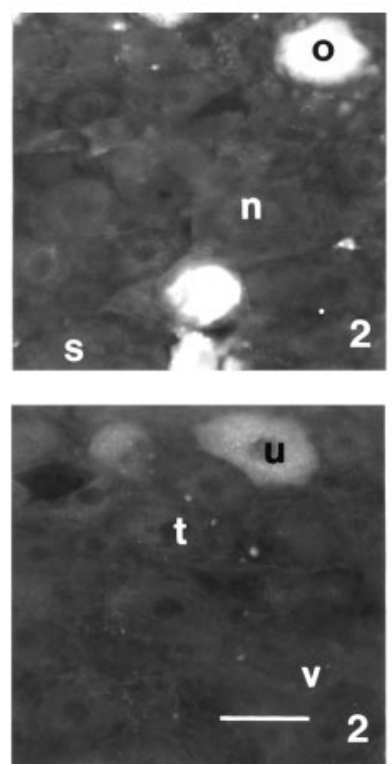

p75
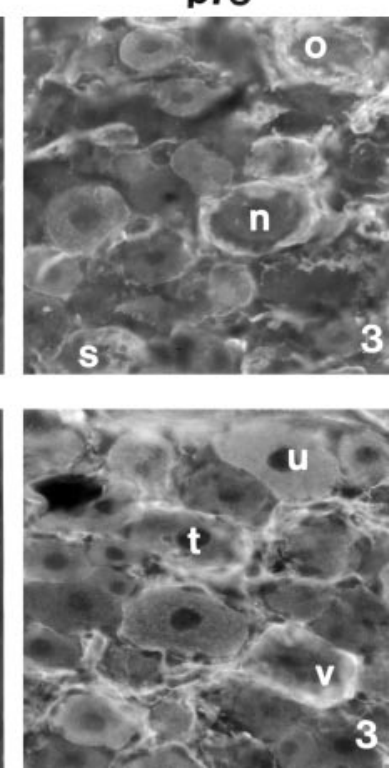

Figure 5. Expression of p75 in satellite cells and neurons 10 weeks after sciatic nerve transection. Perineuronal rings are associated with $\mathrm{p} 75+$ satellite cells around cutaneous somata, whereas p 75 is expressed in muscle rather than cutaneous somata after the lesion. $A$, Cutaneous afferent neurons labeled with FG. B, Muscle afferent neurons labeled with FG. 1, Varicose tyrosine hydroxylase (TH)-positive terminals. 2, FG-labeled neurons. 3, p75 immunoreactivity. Matching letters in the three micrographs indicate the same neuron. $\mathrm{TH}+$ terminals were associated with large-diameter cutaneous neurons surrounded by a multiple layers of p75-positive satellite cells. In contrast, muscle neurons expressed p75 but lacked the ensheathment of p75+ satellite cells. Scale bar: (in B2) $A 1-B 3,50 \mu \mathrm{m}$.

The mean diameter of labeled muscle neurons on the side of the spinal lesion was significantly smaller than on the contralateral side (Fig. $4 B)(p<0.0001)$, and the size distribution showed a clear shift to smaller values (Fig. $3 C$ ). Although the range of cell diameters present was the same, only $11 \%$ of cells were $>40 \mu \mathrm{m}$ in diameter; compare $30-35 \%$ in all other groups $(p<0.001)$. Given the absence of cell loss, these data indicate that atrophy of axotomized muscle afferent neurons is related to the severity of the nerve lesion.

\section{Many muscle but few cutaneous neurons expressed p75 after nerve lesions}

To determine whether the loss of cutaneous neurons was related to the downregulation of p75 in DRG somata (Zhou et al., 1996), sections containing FG+ neurons were stained for $\mathrm{p} 75$. In naive and contralateral uninjured DRGs, $40 \%$ of all neuron profiles ( $n=225$ ) were $\mathrm{p} 75+$, whereas only $14 \%$ (of 121 somata) expressed p75 10 weeks after sciatic transection (Fig. 5) (Zhou et al., 1996). Of 128 sural neurons, $17 \%$ were $p 75+1$ week after cutting and labeling the sural nerve, and 18\% (of 103) were p75+ 10 weeks after sciatic transection (Fig. 5A). These data indicate that cutaneous neurons lost p75 during the first week after axotomy, but the proportion doing so was the same after sciatic transection. This suggests that both p75+ and p75- sural neurons died over time. In contrast, $\sim 90 \%$ of 259 naive and contralateral FGlabeled muscle neurons expressed p75 (Fig. 5B). This was not affected 1 week after axotomy and was reduced by only $15 \% 10$ weeks after sciatic transection (to $75 \% ; n=116 ; p=0.06$ ). Both small and large neurons were $\mathrm{p} 75+$, with a greater proportion of p75 + muscle neurons being of intermediate diameter by 10 weeks after sciatic transection. Despite the persistence of p75 in muscle neurons, the overall reduction in p75 expression reflects

the predominance of cutaneous neurons in the L5 DRG (Baron et al., 1988).

\section{Satellite cells containing p75 surrounded cutaneous but not muscle neurons}

After peripheral nerve lesions, p75 is expressed in satellite cells surrounding largediameter DRG somata (Zhou et al., 1996). Some neurons bearing p75+ rings contained FG transported from the transected sural nerve (Fig. 5A). In contrast, satellite cells surrounding muscle FG+ neurons lacked p75 (Fig. 5B3, cell u). In fact, 80\% of muscle afferent neurons $(n=35)$ lacked satellite cells immunoreactive for glial fibrillary acidic protein (GFAP), and only one muscle neuron was surrounded by a ring of GFAP + cells. Macrophages containing major histocompatibility complex class II (MHC II) were present among the satellite cells around many cutaneous neurons and also around $80 \%$ of muscle FG+ neurons.

\section{Perineuronal rings formed \\ preferentially around cutaneous rather than muscle neurons after sciatic transection}

p75 expression in satellite cells is responsible for the formation of perineuronal rings of ectopic nerve terminals (Walsh et al., 1999) that arise within the lesioned DRG because of local neurotrophin production (Zhou et al., 1999). Varicose perineuronal rings containing tyrosine hydroxylase $(\mathrm{TH})$, calcitonin gene-related peptide (CGRP), galanin (GAL), or synaptophysin (Syn) were present around medium- to large-diameter somata in all sections of ipsilateral DRGs after sciatic or spinal nerve transection 10 weeks previously (Fig. 6) (Hu and McLachlan, 2001). All types of varicose ring were associated with perisomatic p75+ satellite cells.

Notably, rings were formed around only a small proportion of the medium- to large-diameter neurons that were surrounded by p75+ satellite cells. Thus, if proliferating satellite cells can present high neurotrophin concentrations, p75 itself is not enough to trigger the formation of rings around particular neurons.

After sciatic transection, neurons retrogradely labeled both from sural nerve and muscle bore rings that were immunoreactive for all the markers tested (Figs. 5, $7 A-C$ ). These rings were almost all varicose. Of rings formed around sural neurons, $33.2 \pm$ $5.7 \%$ were $\mathrm{TH}+, 27.8 \pm 6.2 \% \mathrm{CGRP}+, 26.8 \pm 3.4 \% \mathrm{GAL}+$, and $30.0 \pm 4.4 \%$ Syn $+(n=5)$. In contrast, $\sim 10$-fold fewer rings of all types formed around muscle afferent neurons after the same lesion $(\mathrm{TH}+, 3.0 \pm 0.3 \%$; CGRP +, $3.4 \pm 0.9 \%$; GAL +, $4.8 \pm$ $0.8 \%$; Syn,$+ 3.2 \pm 0.9 \% ; n=5$ ); similar proportions of muscle neurons bore rings after spinal nerve transection. The total numbers of each type of ring were not significantly different if either skin or muscle neurons had been labeled.

The mean diameter of the rings around sural neurons was $40 \pm 2 \mu \mathrm{m}$ and around muscle afferent neurons was $41 \pm 2 \mu \mathrm{m}$. These terminals lay among the satellite cells that encircled somata that were only slightly larger than the mean diameter of all labeled sural and muscle cells at that time $(\sim 32 \mu \mathrm{m})$ (Fig. 2$)$. This indi- 

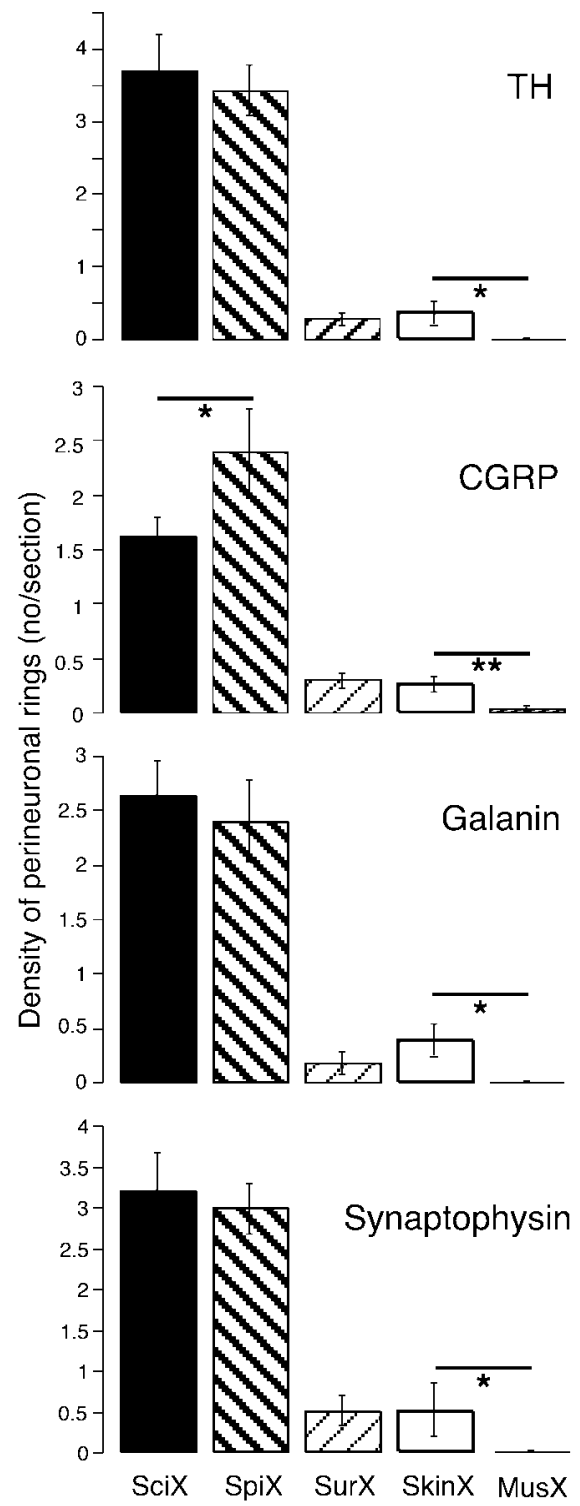

Figure 6. Density of perineuronal rings after different nerve lesions. Histograms show the number of perineuronal rings of terminals per section present in the L5 DRG 10 weeks after various lesions indicated at the bottom of the figure. Rings containing tyrosine hydroxylase (TH), calcitonin gene-related peptide (CGRP), galanin, and synaptophysin are shown for different lesions: SciX, sciatic nerve cut (black; $n=10$ rats); SpiX, spinal nerve cut (left bold hatching; $n=3$ ); SurX, sural nerve cut (right faint hatching; $n=4$ ); SkinX, four skin nerves cut (white; $n=4$ ); MusX, muscle nerves cut (vertical hatching; $n=4$ ). The effects of sural, skin, and muscle nerve transections were all significantly different from sciatic and spinal nerve transections $(p<0.001)$. Asterisks indicate other differences: ${ }^{*} p<0.05$; ${ }^{* *} p<0.01$. Note that there were virtually no rings formed after muscle nerve transection.

cates that medium- to large-diameter cells projecting to either target can accept rings.

The higher number of rings formed around sural neurons might have resulted simply because there were fewer muscle neurons present in the DRG. Ten weeks after sciatic transection, 35 labeled sural neurons remained in each section, whereas there were 10 labeled muscle neurons per section after the same period, as in controls. Thus, one would expect to find 3.5 times more rings around sural neurons than around muscle afferent somata. Instead the number differed by a factor of 10 . This implies a strong tendency for perineuronal rings to form around cutaneous rather than muscle afferent neurons.
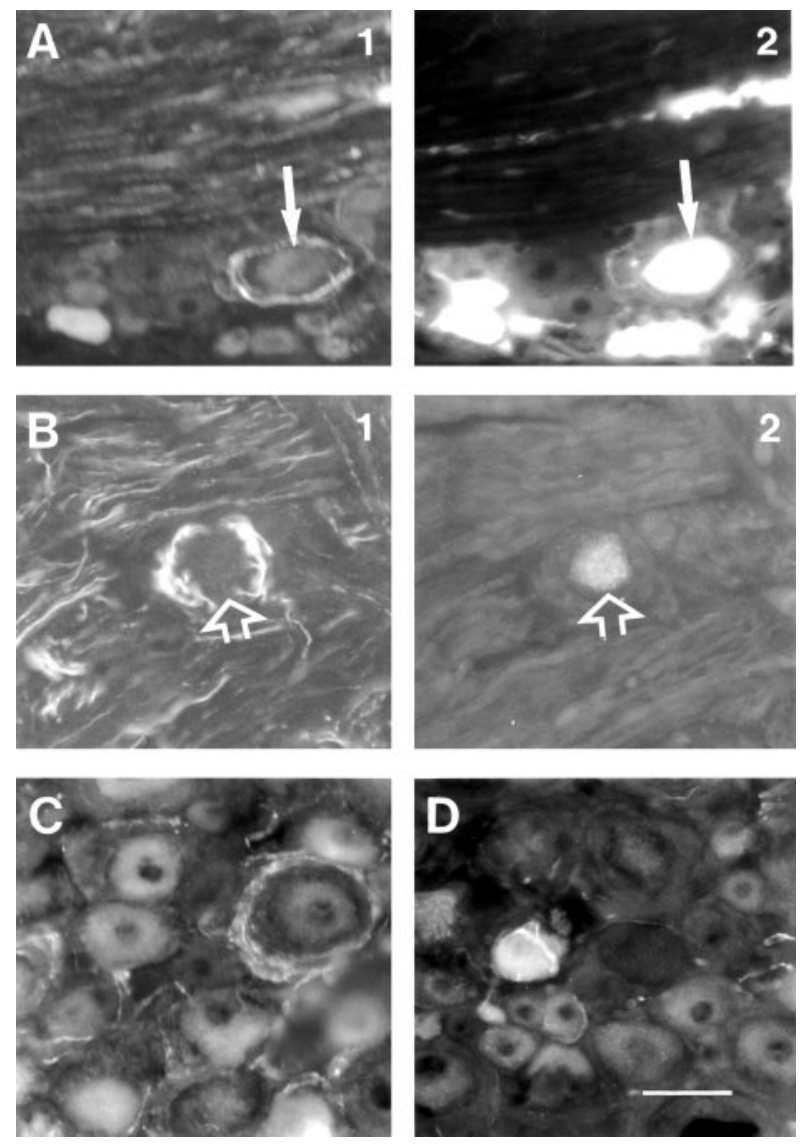

Figure 7. Different types of immunoreactive perineuronal ring structures in DRGs 10 weeks after peripheral nerve transection. $A$, A ring of varicose galanin-positive terminals surrounds a cutaneous afferent neuron (arrows). $B$, A ring of varicose tyrosine hydroxylase-positive terminals surrounds a muscle afferent neuron (arrows). $C$, A ring of varicose synaptophysin-positive terminals. D, A small CGRP + neuron soma surrounded by a coiled smooth CGRP + axon, probably arising from the same cell. $A, C, D$, After sciatic nerve transections; $B$, after spinal nerve transection. Scale bar: (in $D) A-D, 50 \mu \mathrm{m}$.

When only the sural nerve had been cut, there were only 15$20 \%$ of the number of rings of all types present after sciatic or spinal nerve transection, and $\sim 50 \%$ of these were around labeled sural neurons. Thus rings form as readily around axotomized as intact cutaneous neurons.

Perineuronal rings were more likely to form when cutaneous rather than muscle nerves were cut and ligated

To determine whether lesions to cutaneous or muscle neurons were more likely to trigger the formation of perineuronal rings, major nerve trunks in the distal hindlimb that predominantly supplied skin or muscle (see Materials and Methods) were ligated and cut independently, and the DRGs were examined 10 weeks later.

Surprisingly, after transecting three other major cutaneous nerves as well as the sural nerve ( $n=4$ rats), the number of perineuronal rings in L5 DRG was only $10-15 \%$ of the number present after section of the entire sciatic nerve and not significantly different from that after sural transection alone (Figs. 6, 8).

After transection of the nerves supplying the anterior and posterior muscle compartments of the distal hindlimb ( $n=4$ rats), virtually no perineuronal rings were formed (Fig. 6).

After cutaneous nerve lesions, about half of the CGRP + rings were coils rather than varicose terminals, and coils made up the 


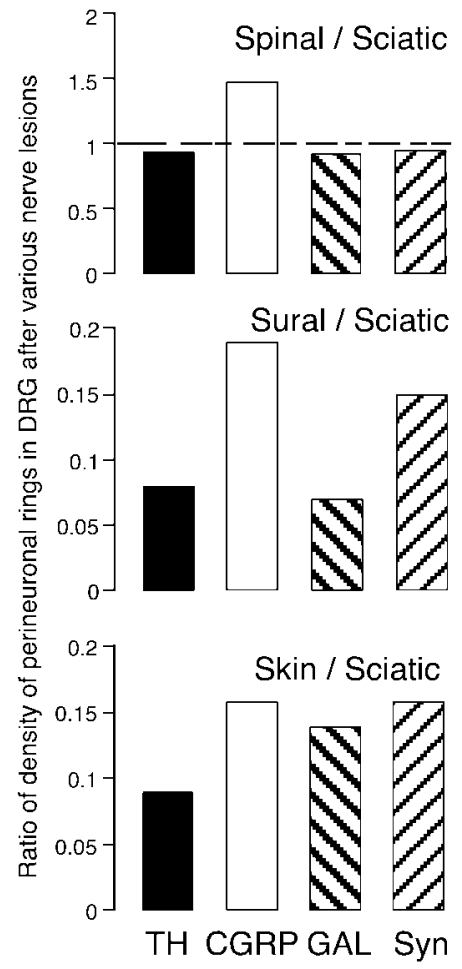

Figure 8. Comparison of the density of perineuronal rings after different nerve lesions. Histograms show the ratios of the density of rings in DRG sections after sciatic, spinal, sural, and skin nerve lesions. Rings containing TH (black), CGRP (white), galanin (GAL, left bold hatching), and synaptophysin (Syn, right hatching). Except for the number of CGRP rings that includes coils ( $\sim 50 \%$ of total in each case), the ratios are similar. The effect of spinal nerve transection was similar to that of sciatic nerve transection. The effects of cutting four skin nerves was almost the same as that of cutting only the sural nerve except for the number of GAL + rings. Syn + rings were more numerous because Syn is present in varicosities of all types.

great majority of the rare CGRP + perineuronal rings detected after muscle nerve lesions (Fig. 7D). Even so, CGRP+ axonal coils were present after muscle nerve lesions at approximately $50 \%$ of the frequency observed when the cutaneous nerves had been cut.

Comparison of the ratios of the densities of rings after different lesions (Fig. 8) shows that the number of perineuronal rings formed was not directly related to the extent of the lesion (see Discussion).

\section{Discussion}

Cutaneous and muscle afferent neurons have been found to differ in their response to peripheral nerve transection in several ways: (1) The small-diameter DRG neurons that progressively die are those that originally supplied skin as their target tissues. As well as axotomy and the loss of target-derived neurotrophins, factor or factors in the perisomatic environment in the DRG contribute to neuronal death. (2) Perineuronal baskets of varicose terminals develop from sprouting sympathetic and sensory axons within the DRG more frequently around cutaneous than muscle neurons. (3) Perineuronal rings are induced after section of cutaneous but not muscle nerves. Some of these findings were related to differences in p75 expression in the two neuron populations.

\section{Loss of cutaneous neurons}

Unbiassed counts of neurons have revealed that no sensory neurons die in the first week after sciatic transection (Tandrup et al., 2000 ), consistent with $\sim 35 \%$ of DRG profiles being retrogradely labeled from the sural nerve (Sittiracha and McLachlan, 1986; Baron et al., 1988). The loss of small sural neurons after 10 weeks was detected by a $30 \%$ decrease in the number of retrogradely labeled somata and the disappearance of neuronal profiles of smaller diameter. It seems unlikely that FG disappeared from neuron somata rather than that the neurons died for several reasons. First, evidence of the gradual loss of small DRG neurons after nerve lesions is available (Tandrup et al., 2000), and the extent of disappearance of FG + sural neurons is consistent with this. Second, sural neuron death was greater when the other sciatic axons were also cut. This did not affect FG turnover in the muscle population over the same period. Third, FG persisted in neurons contralateral to the lesion for 10 weeks after muscle injections. Thus, despite some limitations regarding the precise numbers of dead neurons caused by the profile counting technique, the data provide clear evidence of the selective vulnerability of small cutaneous neurons after nerve lesions.

Apoptotic death of axotomized neurons is thought to follow retrograde signals from the lesion site, the chromatolytic response of the neurons, and the absence of neurotrophic factors supplied from the target tissues (Groves et al., 1999). Because death was more common if the whole sciatic nerve was cut, factors in the environment around axotomized neurons also trigger the death cascade. These might include cytokines such as tumor necrosis factor $\alpha$ (TNF- $\alpha$ ), which is upregulated within the lesioned DRG (Schäfers et al., 2003a), and/or the invasion of immune cells (Hu and McLachlan, 2002).

From their dimensions, the cells that died after axotomy were probably nociceptive neurons that retain their dependence on target-derived neurotrophins in the adult (Lawson, 2002). Despite the production of NGF and other neurotrophins by satellite cells in the lesioned DRG (Zhou et al., 1999), downregulation of neuronal trk receptors limits their utilization (Li et al., 2000). Functionally, progressive death of nociceptive neurons may not exacerbate the initial effect, given the prompt retraction of their central terminals from lamina II (Mannion and Woolf, 2000), but it would certainly reinforce the long-term consequences of modified connectivity within the dorsal horn.

\section{Muscle afferent neurons survive peripheral nerve transection}

Evidence against death of muscle neurons after sciatic or spinal nerve transection depends critically on the constant number of cells labeled from the muscle injections. The number of labeled neurons was remarkably similar between control and operated DRGs, being unaffected by any form of nerve transection. Only $\sim 10$ somata were labeled in each section of L5 DRG, consistent with the relative numbers of cutaneous and muscle neurons (Baron et al., 1988).

p75 was expressed by muscle neurons but lost from sural ones after axon lesions. This may be related to the ability of muscle neurons to survive, consistent with a role for p75 as a neurotrophin receptor (Koliatsos et al., 1991) rather than in cell death signaling (Ferri et al., 2002). However, a subset of muscle neurons becomes hyperexcitable and exhibits the ectopic activity thought to underlie neuropathic pain (Michaelis et al., 2000). p75, as TNF- $\alpha$ receptor type 2 , might be implicated in the enhanced TNF- $\alpha$ sensitivity of muscle sensory neurons (Schäfers et al., 2003b) because intracellular TNF levels are raised after retrograde transport from a distal injury site (Shubayev and Myers, 2001; Schäfers et al., 2003a). The lack of p75 in cutaneous neurons argues against the interaction of TNF- $\alpha$ with TNFR2 as a mediator of neuronal death. 


\section{Preferential formation of perineuronal rings around large cutaneous neurons}

Perineuronal rings were formed preferentially around cutaneous rather than muscle neurons, when the relative sizes of the populations were allowed for. This probably reflects the expression of p75 by proliferated satellite cells associated only with the larger diameter cutaneous population, confirming data from genetically modified mice (Walsh et al., 1999). The presence of MHC $\mathrm{II}+$ macrophages around both cutaneous and muscle neurons rules out a link between these macrophages and the formation of perineuronal rings. The cutaneous neurons are likely to be lowthreshold mechanoreceptors that acquire access to the pain pathways after injury leading to touch-induced allodynia (Mannion and Woolf, 2000). The selective association of $\mathrm{TH}+$ rings with neurons that project to the gracile nucleus suggests these may play a specific role (Ma and Bisby, 1999), although this remains controversial.

\section{Are perineuronal rings formed only if cutaneous nerves are lesioned?}

The failure to generate perineuronal rings after cutting only muscle nerves may be explained by the absence of upregulated p 75 in satellite cells, because axotomy of cutaneous neurons appears to be necessary for this. However, the relationship between the number of rings and the number of lesioned cutaneous afferent axons is not simple. After transecting either four large skin nerves or the sural nerve alone, the number of rings was only $10-15 \%$ of the number after transection of the entire sciatic nerve (Fig. 8). This might result simply because a large proportion of neurons in L5 DRG project in the sural nerve (Baron et al., 1988). However, it does not explain why sciatic nerve transection is so effective unless the formation of rings requires skin and muscle neurons to be lesioned together. After sciatic and spinal nerve transections, the density of rings was approximately proportional to the number of surviving axotomized neurons. It is clear that all the necessary factors for ring formation have yet to be determined.

The present experiments have provided strong evidence that cutaneous nociceptor neurons are selectively vulnerable, not only to axotomy but also to damage to neighboring sensory neurons. This is probably related to downregulation of p75 in cutaneous but not muscle neurons after axotomy. The selective loss of nociceptor neurons triggers changes in connectivity in the dorsal horn leading to abnormal pain signaling (Fields et al., 1998; Mannion and Woolf, 2000). It is less clear whether sensory disturbances depend on the formation of the various types of perineuronal ring. Alternatively they may simply reflect the activation of extraneuronal signals in the lesioned DRG without practical consequences.

\section{References}

Aldskogius H, Risling M (1981) Effects of sciatic neurectomy on neuronal numbers and size distribution in the L7 ganglion of the kitten. Exp Neurol 74:597-604.

Baron R, Jänig W, Kollmann W (1988) Sympathetic and afferent somata projecting in hindlimb nerves and the anatomical organization of the lumbar sympathetic nervous system of the rat. J Comp Neurol 275:460-468.

Ferri CC, Ghasemlou N, Bisby MA, Kawaja MD (2002) Nerve growth factor alters p75 neurotrophin receptor-induced effects in mouse facial motoneurons following axotomy. Brain Res 950:180-185.

Fields HL, Rowbotham M, Baron R (1998) Postherpetic neuralgia: irritable nociceptors and deafferentation. Neurobiol Dis 5:209-227.

Groves MJ, Christopherson T, Giometto B, Scaravilli F (1997) Axotomyinduced apoptosis in adult rat primary sensory neurons. J Neurocytol 26:615-624.
Groves MJ, An SF, Giometto B, Scaravilli F (1999) Inhibition of sensory neuron apoptosis and prevention of loss by NT-3 administration following axotomy. Exp Neurol 155:284-294.

Hökfelt T, Zhang X, Weisenfeld-Hallin Z (1994) Messenger plasticity in primary sensory neurons following axotomy and its functional implications. Trends Neurosci 17:22-30.

Hu P, McLachlan EM (2000) Distinct sprouting responses of sympathetic and peptidergic sensory axons proximal to a sciatic nerve transection in guinea pigs and rats. Neurosci Lett 295:59-63.

Hu P, McLachlan EM (2001) Long-term changes in the distribution of galanin in dorsal root ganglia after sciatic or spinal nerve transection in rats. Neuroscience 103:1059-1071.

Hu P, McLachlan EM (2002) Macrophage and lymphocyte invasion of dorsal root ganglia after peripheral nerve lesions in the rat. Neuroscience 112:23-38.

Jänig W, McLachlan EM (1984) On the fate of sympathetic and sensory neurons projecting into a neuroma of the superficial peroneal nerve in the cat. J Comp Neurol 225:302-311.

Koliatsos VE, Crawford TO, Price DL (1991) Axotomy induces nerve growth factor receptor immunoreactivity in spinal cord motoneurons. Brain Res 549:297-304.

Lawson SN (2002) Phenotype and function of somatic primary afferent nociceptive neurones with C-, Adelta- or Aalpha/beta-fibres. Exp Physiol 87:239-244.

Lekan HA, Chung K, Yoon YW, Chung JM, Coggeshall RE (1997) Loss of dorsal root ganglion cells concomitant with dorsal root axon sprouting following segmental nerve lesion. Neuroscience 81:527-534.

Li L, Zhou XF (2001) Pericellular Griffonia simplicifolia I isolectin B4binding ring structures in the dorsal root ganglia following peripheral nerve injury in rats. J Comp Neurol 439:259-274.

Li L, Deng YS, Zhou XF (2000) Downregulation of TrkA expression in primary sensory neurons after unilateral lumbar spinal nerve transection and some rescuing effects of nerve growth factor infusion. Neurosci Res 38:183-191.

Lisney SJW (1989) Regeneration of unmyelinated axons after injury of mammalian peripheral nerve. Q J Exp Physiol 74:757-784.

Liu CN, Devor M, Waxman SG, Kocsis JD (2002) Subthreshold oscillations induced by spinal nerve injury in dissociated muscle and cutaneous afferents of mouse DRG. J Neurophysiol 87:2009-2017.

Lu X, Richardson PM (1993) Responses of macrophages in rat dorsal root ganglia following peripheral nerve injury. J Neurocytol 22:334-341.

Ma W, Bisby MA (1999) Partial sciatic nerve transection induced tyrosine hydroxylase immunoreactive axon sprouting around both injured and spared dorsal root ganglion neurons which project to the gracile nucleus in middle-aged rats. Neurosci Lett 275:117-120.

Mannion RJ, Woolf CJ (2000) Pain mechanisms and management: a central perspective. Clin J Pain 16:S144-156.

McLachlan EM, Jänig W, Devor M, Michaelis M (1993) Peripheral nerve injury triggers noradrenergic sprouting within dorsal root ganglia. Nature 363:543-546.

McLachlan EM, Keast JR, Bauer M (1994) SP- and CGRP- immunoreactive axons differ in their ability to reinnervate the skin of the rat tail. Neurosci Lett 176:147-151.

Michaelis M, Liu X, Jänig W (2000) Axotomized and intact muscle afferents but no skin afferents develop ongoing discharges of dorsal root origin after peripheral nerve lesion. J Neurosci 20:2742-2748.

Noguchi K, Dubner R, De Leon M, Senba E, Ruda MA (1994) Axotomy induces preprotachykinin gene expression in a subpopulation of dorsal root ganglion neurons. J Neurosci Res 37:596-603.

Ramer MS, Murphy PG, Richardson PM, Bisby MA (1998) Spinal nerve lesion-induced mechanoallodynia and adrenergic sprouting in sensory ganglia are attenuated in interleukin-6 knockout mice. Pain 78:115-121.

Ramer MS, Thompson SWN, McMahon SB (1999) Causes and consequences of sympathetic basket formation in dorsal root ganglia. Pain 6:S111-S120.

Schäfers M, Geis C, Svensson CI, Luo ZD, Sommer C (2003a) Selective increase of tumor necrosis factor-alpha in injured and spared myelinated primary afferents after chronic constrictive injury of rat sciatic nerve. Eur J Neurosci 17:791-804.

Schäfers M, Lee DH, Brors D, Yaksh TL, Sorkin LS (2003b) Increased sensitivity of injured and adjacent uninjured rat primary sensory neurons to 
exogenous tumor necrosis factor- $\alpha$ after spinal nerve ligation. J Neurosci 23:3028-3038.

Shubayev VI, Myers RR (2001) Axonal transport of TNF- $\alpha$ in painful neuropathy: distribution of ligand tracer and TNF receptors. J Neuroimmunol 114:48-56.

Sittiracha T, McLachlan EM (1986) Evaluation of the effects of various additives on retrograde labelling by horseradish peroxidase applied to intact and transected hindlimb nerves of rat and rabbit. Neuroscience 18:763-772.

Tandrup T, Woolf CJ, Coggeshall RE (2000) Delayed loss of small dorsal root ganglion cells after transection of the rat sciatic nerve. J Comp Neurol 422:172-180.

Vestergaard S, Tandrup T, Jakobsen J (1997) Effect of permanent axotomy on number and volume of dorsal root ganglion cell bodies. J Comp Neurol 388:307-312.

Walsh GS, Krol KM, Kawaja MD (1999) Absence of the p75 neurotrophin receptor alters the pattern of sympathosensory sprouting in the trigeminal ganglia of mice overexpressing nerve growth factor. J Neurosci 19:258-273.

Wesselmann U, McLachlan EM (1984) The effect of previous transection on quantitative estimates of the preganglionic neurones projecting in the cervical sympathetic trunk of the guinea pig and the cat made by retrograde labelling of damaged axons by horseradish peroxidase. Neuroscience 13:1299-1309.

Zhou XF, Rush RA, McLachlan EM (1996) Differential expression of the p75 nerve growth factor receptor in glia and neurons of the rat dorsal root ganglia after peripheral nerve transection. J Neurosci 16:2901-2911.

Zhou X-F, Deng Y-S, Chie E, Xue Q, Zhong J-H, McLachlan EM, Rush RA, Xian C (1999) Satellite cell-derived nerve growth factor and neurotrophin-3 are involved in noradrenergic sprouting in the dorsal root ganglia following peripheral nerve injury in the rat. Eur J Neurosci 11:1711-1722. 